\title{
Análise da previsibilidade dos implantes dentários curtos: revisão sistemática da literatura
}

\author{
Analysis of the previsibility of short dental implants: systematic literature \\ review
}

1 Amanda Borges Rodrigues

1 Duglaci Borges De Castro Neto

2 Paulo Cézar Simamoto Júnior

3 Luana Cardoso Cabral luanacardoso29@yahoo.com.br

1 Graduanda/o na Faculdade Morgana Potrich, Curso de Odontologia, Mineiros, GO.

2 Professor na área de oclusão, prótese fixa e materiais odontológicos da Universidade Federal de Uberlândia, Curso de Odontologia, Uberlândia, MG.

3 Professora na área de oclusão, prótese fixa e materiais dentários da Faculdade Morgana Potrich, Curso de Odontologia, Mineiros, GO, Brasil. Doutoranda em Clínica Odontológica Integrada pela Universidade Federal de Uberlândia, Uberlândia, MG.

\section{Resumo}

Reabilitar áreas com reabsorções ósseas severas torna-se um processo complexo, especialmente em regiões posteriores da cavidade oral, por apresentarem estruturas anatômicas nobres que podem ser lesionadas com a colocação de implantes com comprimento convencional. Os implantes curtos são opções de tratamento para esses casos limitantes. Contudo, há poucas evidências de boa qualidade quanto ao uso dessas reabilitações. Nesse contexto, o objetivo do estudo foi analisar a previsibilidade dos implantes curtos por meio da revisão sistemática de literatura. O delineamento do estudo seguiu as diretrizes do Preferred Reporting Items for Systematic Reviews and Meta-Analyses (PRISMA) Checklist. As bases de dados analisadas foram PubMed, Scielo e Biblioteca Virtual em Saúde. Um total de 149 estudos foram identificados. Após a remoção dos estudos duplicados e leitura dos títulos e resumos, 50 foram lidos na íntegra e 7 foram incluídos. Desse total, 1.807 pacientes foram avaliados; 340 receberam apenas implantes curtos e 1.467 receberam curtos e longos. Os implantes instalados totalizaram em 3.906 (908 curtos e 2998 longos). As taxas de sobrevivência dos implantes curtos variaram entre $95 \%$ a $98 \%$ em um período de acompanhamento médio de 5 anos. Conclui-se que os implantes curtos se mostram eficazes e previsíveis no tratamento reabilitador de rebordos residuais com aproximadamente $5 \mathrm{~mm}$. As complicações, quando presentes, são: perda óssea marginal, peri-implantite, parestesia do nervo alveolar inferior, afrouxamento do parafuso e fratura do revestimento cerâmico.

\section{Palavras-chave:}

Implantes dentários. Prótese parcial fixa. Complicações pós-operatórias.

\begin{abstract}
Rehabilitate areas with severe bone resorption is a complex process, especially in posterior regions of the oral cavity. This fact is justified by the presence of noble anatomical structures, which can be injured by placing conventional length implants. Short implants are treatment options for these limiting cases. However, there is little good quality evidence regarding the use of these rehabilitations. In this context, the study aimed to analyze the predictability of short implants through a systematic review. The study design followed the guidelines of the Preferred Reporting Items for Systematic Reviews and Meta-Analyses (PRISMA) Checklist. The databases analyzed were PubMed, Scielo and Virtual Health Library. A total of 149 studies were identified. After removing duplicates and reading the titles and abstracts, 50 were read in full and 7 were included. Thus, 1,807 patients were evaluated; 340 received only short implants and 1,467 received short and long implants. The installed implants totalized 3,906 (908 short and 2998 long). Survival rates for short implants ranged from $95 \%$ to $98 \%$ over an average follow-up period of 5 years. It is concluded that the short implants are effective and predictable in the rehabilitation treatment of residual edge with approximately $5 \mathrm{~mm}$. Complications, when present, are: marginal bone loss, peri-implantitis, paresthesia of the lower alveolar nerve, loosening of the screw and fracture of the ceramic lining..
\end{abstract}

\section{Keywords:}

Dental implants. Fixed partial denture. Postoperative complications.

\section{Como você deve citar?}

RODRIGUES, Amanda Borges et al. Análise da previsibilidade dos implantes dentários curtos: revisão sistemática da literatura. Cadernos UniFOA, Volta Redonda (RJ), v. 16, n. 46, p. 1-9, ago, 2021. 


\section{INTRODUÇÃO}

A qualidade de vida em adultos pode ser afetada pela perda dentária e, consequentemente, comprometimento da função oral, influenciando na autoestima (UEHERA; TAKAOKA; ITO, 2004). 0 uso de implantes para reabilitar regiões edêntulas tem aumentado nos últimos 30 anos, devido à alta taxa de sucesso, previsibilidade nos procedimentos e índice de complicações relativamente baixo (GONZALEZ et al., 2014; HONG \& OH, 2017).

Durante o planejamento, várias condições necessitam ser avaliadas como a saúde bucal, ausência de alteração oral e distúrbios sistêmicos, tabagismo, quantidade de mucosa queratinizada, espaço interoclusal e também volume ósseo para instalação e manutenção dos implantes (GOMES et al., 2008).

Quando o volume ósseo não é suficiente para utilização dos implantes com comprimento padrão, diferentes soluções estão disponíveis, como enxertos ósseos, levantamento do seio maxilar, distração osteogênica e transposição do nervo alveolar inferior. Contudo, todos esses procedimentos envolvem tempo de cicatrização prolongado, maior morbidade e custo elevado, por se tratar de cirurgias adicionais no processo reabilitador (NISAND \& RENOUARD, 2014). Os implantes curtos ( $<10 \mathrm{~mm}$ ) foram introduzidos como uma alternativa de tratamento para essas situações limitantes, oferecendo vantagens cirúrgicas (ATIEH et al., 2012; CHRCANOVIC; ALBREKTSSON; WENNERBERG, 2016). Alguns estudos reportam resultados clínicos favoráveis com taxa de sucesso variando entre $92 \%$ a $98 \%$ (LOPS et al., 2012; ANITUA et al., 2014).

No entanto, essa modalidade de tratamento, possui algumas limitações, como relação coroa-implante desfavorável, estética reduzida em maxila atrófica anterior e risco relativo de perda precoce de implantes. Essas condições clínicas são consideradas fatores de risco importantes que podem comprometer a longevidade dos implantes com um menor comprimento (ANNIBALI et al., 2012; PALACIOS et al., 2018).

Há pouca evidência de boa qualidade quanto às vantagens do uso de implantes dentários curtos, havendo bastante controvérsias sobre sua longevidade, devido à diminuição do contato osso-implante. Além disso, a disponibilidade dessas evidências, algumas vezes, são confusas (ANITUA et al., 2014; SHAH, 2015). Nesse contexto, o objetivo do estudo foi analisar a previsibilidade dos implantes curtos por meio da revisão sistemática de literatura.

\section{MATERIAIS E MÉTODOS}

A revisão de literatura foi estruturada de acordo com o Preferred Reporting Items for Systematic Reviews and Meta-Analyses (PRISMA) Checklist (MOHER et al., 2015).

\section{Critérios de Elegibilidade}

Os estudos que reuniram os seguintes critérios foram incluídos: trabalhos prospectivos, retrospectivos e estudos clínicos randomizados que avaliaram clinicamente e radiograficamente os implantes curtos com suas respectivas reabilitações em um período de acompanhamento mínimo de 6 meses. Estudos que apresentaram um dos critérios seguintes foram excluídos: análise de implantes curtos em reabilitações totais, estudos em animais ou laboratoriais, relatos de casos ou série de casos, revisões de literatura, resumos de conferências e cartas ao editor. 


\section{Estratégia de Busca}

A questão da pesquisa foi elaborada seguindo o acrônimo PECO (população/problema, exposição, comparação e desfechos). 0 problema $(P)$ foi área com reabsorção óssea severa; a exposição (E) implantes curtos comparados (C) com implantes longos; e o desfecho (O) foram taxa de sobrevivência dos implantes, quantidade de perda óssea marginal e presença de complicações biológicas e protéticas.

As bases de dados analisadas foram PubMed, Scielo e Biblioteca Virtual da Saúde. Os termos associados para a busca incluíram: "Short implant", "Dental Implants", "Dental Implantation, Endosseus", "Bone-implant Interface" e "Dental implants, single tooth". Após a remoção dos estudos duplicados, a análise se dividiu em três etapas: na primeira, dois revisores independentes (ABR e DBCN) realizaram a leitura dos títulos. Aqueles trabalhos potencialmente relevantes foram considerados para as etapas seguintes. Posteriormente, os resumos foram lidos pelos mesmos revisores (ABR e DBCN). Finalmente, os estudos da etapa três foram analisados na íntegra. Aqueles que apresentaram os critérios de elegibilidade foram incluídos. É importante ressaltar que, nessa etapa, para inclusão, deveria haver concordância entre os revisores. Nos casos de discordância, um terceiro revisor foi recrutado (LCC).

\section{Extração dos dados}

Os seguintes dados foram registrados: autor/ano, número de pacientes, idade média, comprimento dos implantes, diâmetro, período de acompanhamento, complicações e taxa de sobrevivência.

\section{RESULTADOS}

O fluxograma descrevendo a pesquisa e a seleção com o número de artigos incluídos e excluídos é mostrado na Figura 1 . 0 levantamento bibliográfico identificou 149 referências. Após a remoção das referências duplicadas, 147 foram selecionadas para triagem de títulos e resumos. Em seguida, 50 artigos foram selecionados para leitura do texto completo. Após a aplicação dos critérios de elegibilidade, 7 artigos apresentaram os critérios de inclusão para a síntese qualitativa.

Um total de 1.807 pacientes foram avaliados. Desses, 340 receberam apenas implantes curtos e 1.467 receberam curtos e longos. 0 período médio de acompanhamento foi de 5 anos (período mínimo: 6 meses e período máximo: 10 anos). Os implantes instalados totalizaram em 3.906 (908 curtos e 2.998 longos). 0 diâmetro variou entre 3,0 a 5,0 mm. A taxa de sobrevivência observada nos implantes curtos foi de $95 \%$ a $98,7 \%$. As complicações prevalentes incluíram peri-implantite, reabsorções ósseas marginais, afrouxamento do parafuso, fratura do revestimento cerâmico e parestesia transitória do nervo alveolar inferior (Tabela 1). 
Figura 1. Fluxograma da pesquisa literária e critérios de seleção

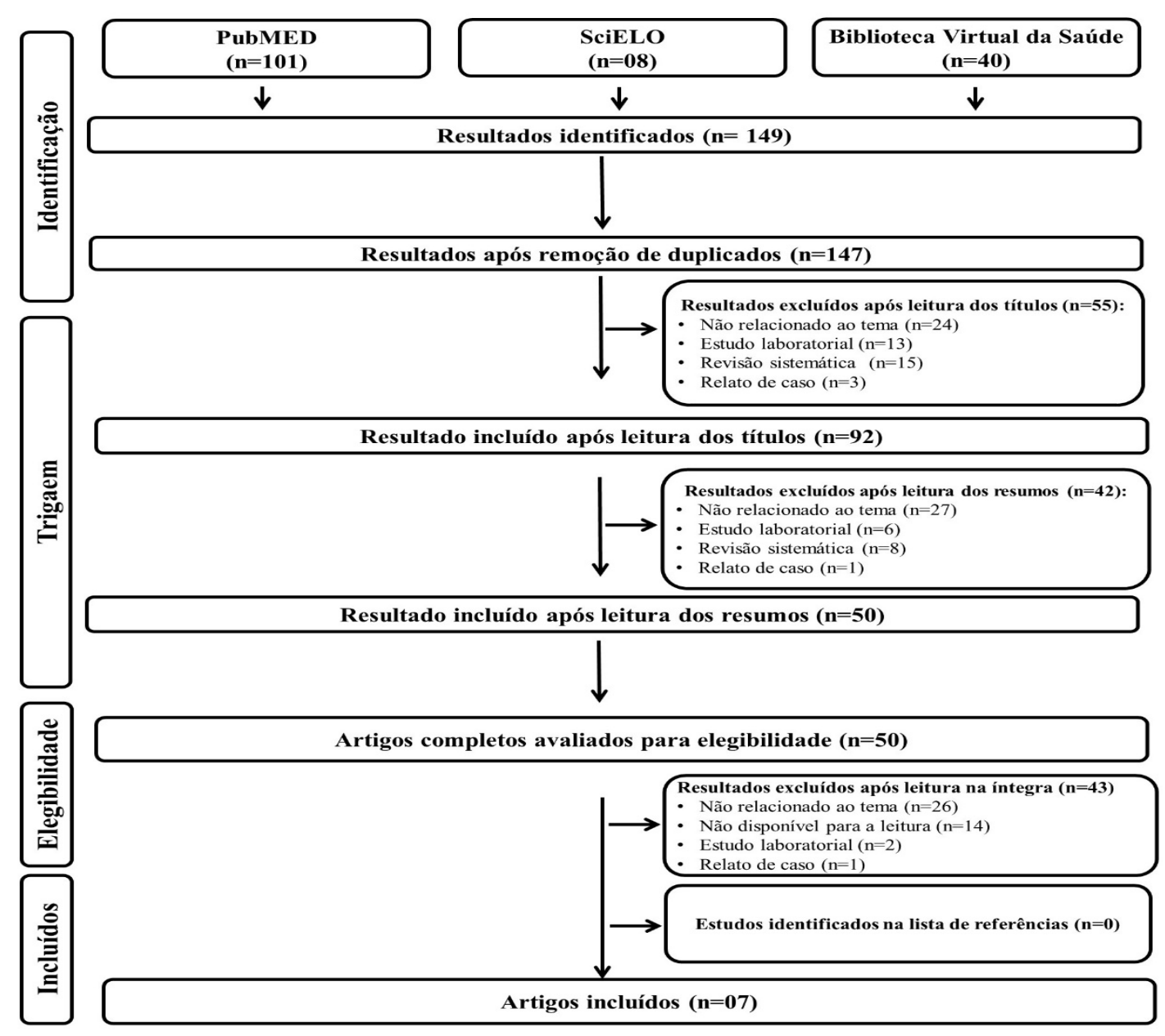

Fonte: autoria própria (2020). 
Tabela 1: Características dos estudos incluídos $(n=7)$

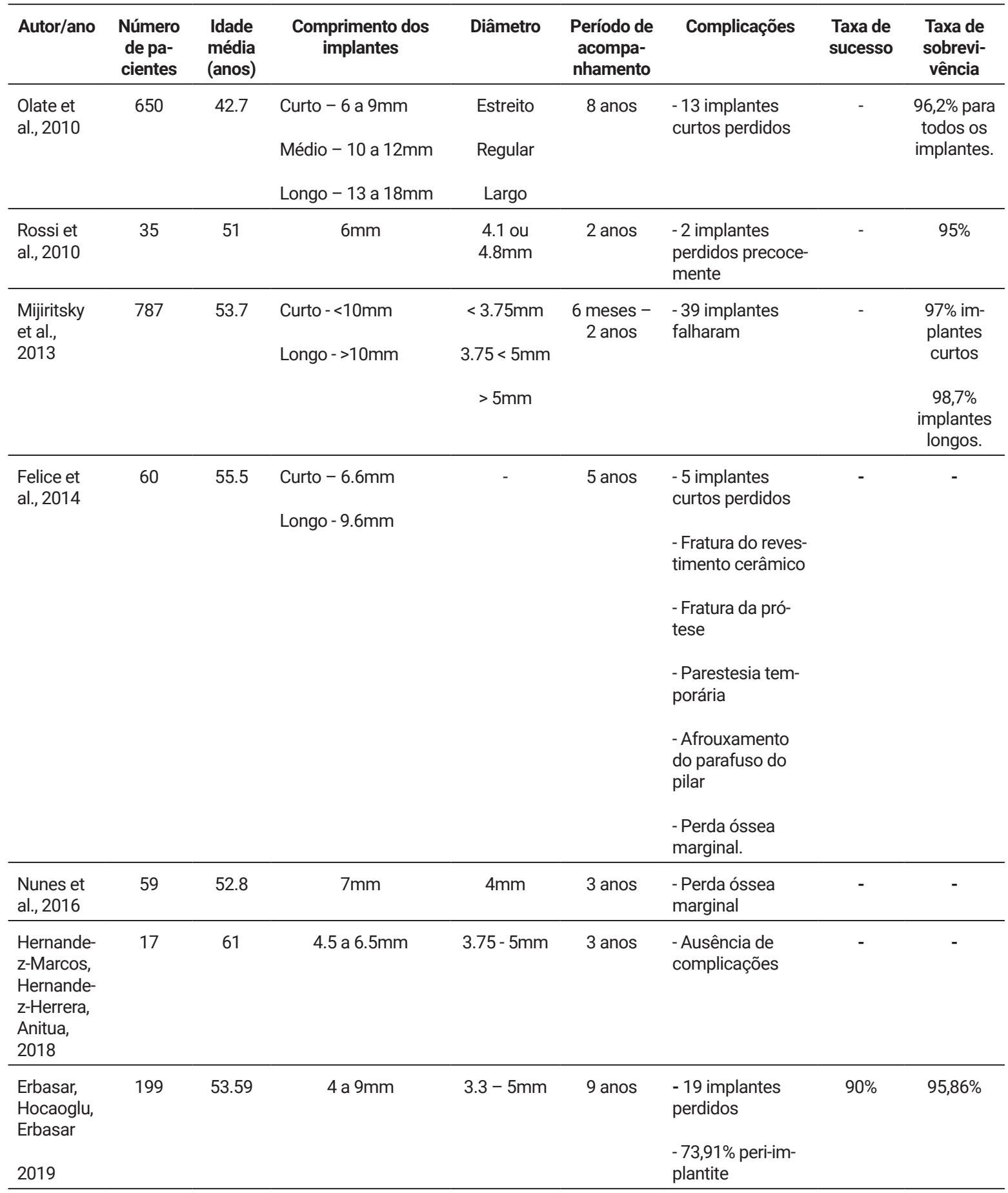

Fonte: autoria própria (2020).

\section{DISCUSSÃO}

A escolha do comprimento do implante em relação à qualidade e quantidade óssea disponível é um fator crítico para o sucesso dessa terapêutica (LEE et al., 2012; LEMOS et al., 2016). O presente estudo revisou os parâmetros clínicos e radiográficos de reabilitações com implantes curtos, a fim de determinar a previsibilidade e longevidade desse tratamento. 
Ao analisar a taxa de sobrevivência, que se baseia na presença do implante em função, pode-se notar que não houve diferença estatisticamente significativa entre os implantes curtos e os implantes com comprimento convencional instalados em sítios enxertados. A taxa variou entre $95 \%$ a $98,7 \%$ (OLATE et al., 2010; MIJIRITSKY et al., 2013; ROSSI et al., 2010; HERNÁNDEZ-MARCOS; HERNÁNDEZ-HERRERA; ANITUA, 2018). Esses achados possivelmente estão relacionados às recentes melhorias mecânicas e morfológicas da superfície do implante. 0 aumento do diâmetro permite um maior contato entre osso e implante, favorecendo assim uma melhor distribuição da força, proporcionando estabilidade (MIJIRITSKY et al., 2013). 0 diâmetro dos implantes desta revisão variou entre $3,3 \mathrm{~mm}$ a $6 \mathrm{~mm}$. A escolha de implantes com superfícies modificadas também garante maiores taxas de sucesso dos implantes curtos (OMRAN et al., 2015; ATIEH et al., 2012). Os diferentes tratamentos de superfície aplicados nos implantes dos estudos incluídos foram: uso de cristais de fosfato em nano escala (FELICE et al., 2014) e superfícies nanotexturizadas (NUNES et al., 2016).

Além da análise das taxas de sobrevivência e sucesso, ao planejar reabilitações orais, é importante avaliar a presença de comorbidades. As falhas nos implantes dentários podem ser subdivididas em falhas precoces e tardias. A primeira associa-se com osseointegração insuficiente. Já a segunda, está relacionada com a microbiota oral, hábitos parafuncionais e diferentes formas de reabilitação, como quantidade e angulação do implante ou pilar e variáveis protéticas (CHRCANOVIC; ALBREKTSSON; WENNERBERG, 2016; ALSAADI et al., 2007; CHRCANOVIC; ALBREKTSSON; WENNERBERG, 2014). As alterações mais comuns em reabilitações implato-retidas são complicações biológicas (perfuração ou infecção sinusal, mucosite periimplantar e peri-implantite) e mecânicas (afrouxamento do parafuso, fratura do parafuso, fratura da coroa ou deslocamento da coroa protética) (YAN et al., 2019). Na presente revisão, as complicações mencionadas foram perda dos implantes (OLATE et al., 2010; ROSSI et al., 2010), peri-implantite (ERBASAR; HOCAOGLU; ERBASAR, 2019), perda óssea marginal (FELICE et al., 2014; NUNES et al., 2016), afrouxamento do parafuso, fratura do revestimento cerâmico e parestesia temporária do nervo alveolar inferior (FELICE et al., 2014).

Dos implantes curtos avaliados, aproximadamente 4,2\% foram perdidos. Esses dados relacionam-se com fatores determinantes, como tabagismo, periimplantite, sobrecarga inicial, qualidade óssea e presença de hábito parafuncional (OLATE et al., 2010). O calor e os subprodutos tóxicos do tabaco influenciam negativamente na cicatrização do tecido, afetando a osseointegração e, portanto, na sobrevivência do implante (NAZEER et al., 2020). Além do tabagismo, a perda também está relacionada à presença de patógenos peri-implantares. A maior parte das doenças peri-implantares tem como fator etiológico o acúmulo de biofilmes microbianos, afetando as estruturas ao redor do implante, que são mucosas e osso alveolar causando reações inflamatórias locais. Para o diagnóstico, deve-se atentar ao sangramento durante a sondagem e aos achados radiográficos (FELICE et al., 2014).

O sucesso do implante após o carregamento é considerado quando há uma observação de perda óssea marginal menor que 0,2 milímetros por ano, processo denominado de saucerização (CONSOLARO et al., 2010). No estudo de Felice et al. (2014) observou-se uma média de $2 \mathrm{~mm}$ de diminuição do tecido duro, após cinco anos de acompanhamento. Essa perda óssea marginal a longo prazo pode ocorrer devido à presença de doenças peri-implantares, sobrecarga oclusal ou relaciona-se à proporção coroa-implante (C/I) (NUNES et al., 2016).

A proporção clínica $\mathrm{C} / \mathrm{I}$ é definida como a relação da altura clínica da coroa (a distância do aspecto mais coronal da restauração para o contato mais coronal osso/implante) e a altura clínica do implante (porção do implante inserido dentro do osso alveolar) (BLANES et al., 2007). 0 aumento dessa relação pode atuar como um cantilever vertical, levando à perda óssea crestal e falha do implante (JAIN et al., 2016). É importante ressaltar que a proporção coroa-implante não teve influência significativa na perda óssea marginal dos implantes curtos avaliados (NUNES et al., 2016). Esse dado pode estar associado com a esplintagem das coroas protéticas. A colocação de implantes esplintados aumenta a área funcio- 
nal da superfície de suporte e transmite menos força para a prótese, os parafusos do pilar e a interface óssea do implante (JAIN et al., 2016).

Além dos aspectos mencionados, o afrouxamento ou fratura do pilar é a falha protética que ocorre com maior frequência nas reabilitações com próteses implanto-suportadas (FELICE et al., 2014). A conexão protética é um dos principais fatores etiológicos e a falta de estabilidade entre implante e pilar ocasiona no afrouxamento ou fratura do parafuso ou pilar (MICHALAKIS et al., 2014). Além da conexão, a sobrecarga oclusal, aumento do comprimento e diminuição do diâmetro dos implantes e composição da liga também aumenta a probabilidade de afrouxamento do parafuso e, por consequência, fratura do implante (CHRCANOVIC; ALBREKTSSON; WENNERBERG, 2014).

Outra complicação observada nos estudos incluídos foi a parestesia transitória (FELICE et al., 2014). A prevalência da lesão ao nervo alveolar inferior pode variar entre $3 \%$ a $14 \%$ de forma transitória e $4 \%$, permanente (DANNAN; ALKATTAN; JACKWSKI, 2013). 0 estudo de Garcia-Ochoa et al. (2020) revelou que complicações sensoriais podem afetar os dentes e/ou lábios/pele e relatou sensibilidade reduzida do nervo alveolar inferior imediatamente após a cirurgia em todos os pacientes que foram analisados. Em geral, eles relataram que as sensações de dormência diminuíram durante o acompanhamento na grande maioria dos casos (GARCIA-OCHOA et al., 2020). Com o diagnóstico de parestesia, é importante acompanhar o paciente durante duas a três semanas, sendo normalmente a recuperação total de dois a três meses.

\section{CONCLUSÃO}

O presente estudo conclui que os implantes curtos se mostram eficazes e previsíveis no tratamento reabilitador de rebordos residuais com, aproximadamente, $5 \mathrm{~mm}$. As complicações, quando presentes, são: perda óssea marginal, peri-implantite, parestesia do nervo alveolar inferior, afrouxamento do parafuso e fratura do revestimento cerâmico.

\section{REFERÊNCIAS}

ALSAADI, G.; QUIRYNEN, M.; KOMÁREK, A.; VAN STEENBERGHE, D. Impact of local and systemic factors on the incidence of oral implant failures, up to abutment connection. J Clin Periodontol, v. 34, p. 610-617, 2007.

ANNIBALI, S.; CRISTALLI, M. P.; DELL'AQUILA, D.; BIGNOZZI, I.; LA MONACA, G.; PILLONI, A. Short Dental Implants: A Systematic Review. J Dent Res, v. 91, p. 25-32, 2012.

ANITUA, E.; PINAS, L.; BEGONA, L.; ORIVE, G. Long-term retrospective evaluation of short implants in the posterior areas: clinical results after 10-12 years. J Clin Periodontol, v. 41, p. 404-411, 2014.

ATIEH, M. A.; ZADEH, H.; STANFORD, C. M.; COOPER, L. F. Survival of short dental implants for treatment of posterior partial edentulism: a systematic review. Int J Oral Maxillofac Implants, v. 27, p. 1323-1331, 2012.

BLANES, R.J.; BERNARD, J.P.; BLANES, Z.M.; BELSER, U.C. A 10-year prospective study of ITI dental implants placed in the posterior region: Clinical and radiographic results. Clin Oral Implants Res, v. 18, p. 699-706, 2007.

CHRCANOVIC, B. R.; ALBREKTSSON, T.; WENNERBERG, A. Reasons for failures of oral implants. J Oral Rehabil, v. 41, p. 443-476, 2014. 
CHRCANOVIC, B. R.; ALBREKTSSON, T.; WENNERBERG, A. Turned versus anodised dental implants: a meta-analysis. J Oral Rehabil, v. 43, p. 716-728, 2016.

CONSOLARO, A.; DE CARVALHO, R. S.; FRANCISCHONE JUNIOR, C. E.; CONSOLARO, M. F. M. O.; FRANCISCHONE, C. E. Saucerization of osseointegrated implants and planning of simultaneous orthodontic clinical cases. Dental Press J Orthod, v. 15, p. 19-30, 2010.

DANNAN, A.; ALKATTAN, F.; JACKOWSKI, J. Altered Sensations of the Inferior Alveolar Nerve after Dental Implant Surgery: a Retrospective Study. Dentistry, v. 13, p. 2161-1122, 2013.

ERBASAR, G. N. H.; HOCAOĞLU, T. P.; ERBASAR, R. C. Risk factors associated with short dental implant success: a long-term retrospective evaluation of patients followed up for up to 9 years. Braz. Oral Res, v. 33, p. $1-10,2019$.

FELICE, P.; CANNIZZARO, G.; BARAUSSE, C.; PISTILLI, R.; ESPOSITO, M. Short implants versus longer implants in vertically augmented posterior mandibles: A randomised controlled trial with 5year after loading follow-up. Eur J Oral Implantol, v.7 p. 359-369, 2014.

GARCIA-OCHOA, P.; GONZÁLEZ, F. P.; MORENO, A. N.; LABRADOR, L. S.; BRINKMANN, J. C. B.; GONZÁLEZ, J. M. M.; MARTÍNEZ, J. L. Q. Complications associated with inferior alveolar nerve reposition technique for simultaneous implant-based rehabilitation of atrophic mandibles. A systematic Literature review. J Stomatol Oral Maxillofac Surg, v. 121, p. 390-396, 2020.

GOMES, E. A.; ASSUNÇÃO, W. G.; COSTA, O. S.; DELBEN, J. A.; BARÃO V. A. R.; TABATA, L. F. Aspectos clínicos relevantes no planejamento cirúrgico-protético em implantodontia. Salusvita, v.27, p. 111-124, 2008.

GONZALEZ, S.; TUAN M. C.; AHN, K. M.; NOWZARI, H. Crestal approach for maxillary sinus augmentation in patients with $\leq 4 \mathrm{~mm}$ of residual alveolar bone. Clin Implant Dent Relat Res, v. 16, p. 827-835, 2014.

HERNÁNDEZ-MARCOS, G.; HERNÁNDEZ-HERRERA, M.; ANITUA, E. Marginal bone loss around short dental implants restored at implant level and with transmucosal abutment: A retrospective study. Int $\mathbf{J}$ Oral Maxillofac Implants, v. 33, p. 1362-1367, 2018.

HONG, F. G. K.; OH J. H. Recent advances in dental implants. Maxillofac Plast Reconstr Surg, v. 39, p. 1-10, 2017.

JAIN, N.; GULATI, M.; GARG, M.; PATHAK, C.J. Short implants: New Horizon in Implant Dentistry. Clin Diagn Res, v. 10, p. 14-17, 2016.

LEE, K. J.; KIM, Y. G.; PARK, J. W.; LEE, J. M.; SUH, J. Y. Influence of crown-to-implant ratio on periimplant marginal bone loss in the posterior region: a five-year retrospective study. J Periodontal Implant Sci, v. 42, p. 231-236, 2012.

LEMOS, C. A.; FERRO-ALVES, M. L.; OKAMOTO, R.; MENDONÇA, M. R.; PELLIZZER EP. Short dental implants versus standard dental implants placed in the posterior jaws: A systematic review and meta-analysis. $\mathbf{J}$ Dent, v. 47, p. 8-17, 2016.

LOPS, D.; BRESSAN, E.; PISONI, G.; CEA, N.; CORAZZA, B.; ROMEO, E. Short implants in partially edentuolous maxillae and mandibles: a 10 to 20 years retrospective evaluation. Int J Dent, 351793, 2012. 
MICHALAKIS, K. X.; CALVANI, P.; MUFTU, S.; PISSIOTIS, A.; HIRAYAMA, H. The Effect of Different ImplantAbutment Connection son Screw Joint Stability. Journal of Oral Implantology, v.40, p. 146-152, 2014.

MIJIRITSKY, E.; MAZOR, Z.; LOREAN, A.; LEVIN, L. Implant Diameter and Length Influence on Survival: Interim Results During the First 2 Years of Function of Implants by a Single Manufacture. Implant Dent, v. 22, p. 394-398, 2013.

MOHER, D.; LIBERATI, A.; TETZLAFF, J.; ALTMAN, D. G. Preferred reporting items for systematic reviews and meta-analyses: The PRISMA Statement. PLoS Med 6(6): e1000097.

NAZEER, J.; SINGH, R.; SURI P.; MOUNESHKUMAR, C. D.; BHARDWAJ, S.; IQUBAL, A.; DINESH. Evaluation of marginal bone loss around dental implants in cigarette smokers and nonsmokers. A comparative study. J Family Med Prim Care, v. 9, p. 729-734, 2020.

NISAND, D.; RENOUARD, F. Short implant in limited bone volume. Periodontol, v. 66, p. 72-96, 2014.

NUNES, M.; ALMEIDA, R. F.; FELINO, A. C.; MALO, P.; NOBRE, M. A. The influence of crown-to implant ratio on short implant marginal bone loss. Int J Oral Maxillofac Implants, v. 31, p. 1156-1163, 2016.

OLATE, S.; LYRIO, M. C. N.; MORAES, M.; MAZZONETTO, R.; MOREIRA, R. W. F. Influence of diameter and length of implant on early dental implant failure. J Oral Maxillofac Surg, v. 68, p. 414-419, 2010.

OMRAN, M. T. A.; MILEY, D. D.; MCLEOD, D. E.; GARCIA, M. N. Retrospective Assessment of survival rate for short endosseous dental implants. Implant Dent, v. 24, p. 185-191, 2015.

PALACIOS, J. A. V.; GARCIA, J. J.; CARAMÊS, J. M. M.; QUIRYNEN, M.; MARQUES, D. N. S. Short implants versus bone grafting and standard-length implants placement: a systematic review. Clin Oral Investig, v. 22 , p. $69-80,2018$.

ROSSI, F.; RICCI, E.; MARCHETTI, C.; LANG, N. P.; BOTTICELLI, D. Early loading of single crowns supported by 6 -mm-long implants with a moderately rough surface: a prospective 2-year follow-up cohort study. Clin Oral Implants Res, v. 21, p. 937-943, 2010.

SHAH, A. K. Short implants-When, where and how? J Int Clin Dent Res Organ, v.7, p. 132-137, 2015.

UEHERA, T.; TAKAOKA, K.; ITO, K. Histological evidence of osseointegration in human retrieved fractured hydroxyapatite-coated screw type implants: a case report. Clin Oral Implants Res, v. 15, p. 540-545, 2004.

YAN, Q. I.; WU, X.; SU, M.; HUA, F.; SHI, B. Short implants ( $\leq 6 \mathrm{~mm}$ ) versus longer implants with sinus floor elevation in atrophic posterior maxilla: a systematic review and meta-analysis. BMJ Open, v. 9, p. 1-12, 2019. 